\title{
Factores pronósticos, evolución y mortalidad en el adulto inmunocompetente hospitalizado por neumonía neumocócica adquirida en la comunidad
}

\author{
Fernando Saldías P1 , Paola Viviani G ${ }^{2}$, D ahiana Pulgar Ba, \\ Francisco Valenzuela $F^{a}$, Sebastián Paredes $E^{a}$, \\ O rlando Díaz P1. \\ Prognostic factors and mortality \\ in immunocompetent adult patients \\ hospitalized with community-acquired \\ pneumococcal pneumonia
}

Background: Streptococcus pneumoniae is the main cause of community-acquired pneumonia in adults. Aim: To describe baseline characteristics, risk factors and clinical outcomes of adult patients hospitalized with pneumococcal pneumonia. Material and methods: Prospective study of adult patients admitted for a community acquired pneumonia in a clinical hospital. Immune deficient patients and those with a history of a recent hospitalization, were excluded. Results: One hundred fifty one immunocompetent patients, aged 16 to 92 years, 58\% males, were studied. Seventy five percent had other diseases, $26 \%$ were admitted to the intensive care unit and 9\% needed mechanical ventilation. There were no differences in clinical features, ICU admission or hospital length of stay among bacteremic and nonbacteremic patients. Thirty days lethality for bacteremic and nonbacteremic patients, was $10.9 \%$ and $11.5 \%$, respectively. The predictive values for lethality of Fine pneumonia severity index and CURB-65 (Confusion, Urea nitrogen, Respiratory rate, Blood pressure, 65 years of age and older) had an area under the ROC curve of 0.8 and 0.69 , respectively. Multivariate analysis disclosed blood urea nitrogen over $30 \mathrm{mg} /$ $\mathrm{dL}$ (odds ratio (OR), 6.8), need for mechanical ventilation (OR, 7.4) and diastolic blood pressure below $50 \mathrm{mmHg}(\mathrm{OR}, 3.9)$, as significant independent predictors of death. Conclusions: Pneumococcal pneumonia was associated with a substancial rate of complications and mortality. Clinical presentation and outcome did not differ significantly among patients with and without bacteremia (Rev Méd Chile 2009; 137: 1545-52).

(Key words: Bacteremia; Pneumonia, bacterial; Streptococcus pneumoniae)

Recibido el 15 de julio, 2009. Aprobado el 4 de noviembre, 2009.

${ }_{1}^{1}$ Departamentos de Enfermedades Respiratorias y ${ }^{2}$ Salud Pública, Facultad de Medicina, Pontificia Universidad Católica de Chile, Santiago de Chile.

anterno de Medicina, Facultad de Medicina, Pontificia Universidad Católica de Chile.

Correspondencia a: Dr. Fernando Saldías P. Departamento de Enfermedades Respiratorias. Pontificia Universidad Católica de Chile. Marcoleta 350, Santiago, Chile. Teléfonos: (562) 6331541 - (562) 3543242. Fax: (562) 6335255.

E mail: fsaldias@med.puc.cl 
$\mathrm{E}^{\mathrm{n}}$ las últimas décadas ha aumentado la incidencia de las infecciones neumocócicas en el adulto, y a pesar de la amplia disponibilidad de antibioterapia eficaz, siguen ocasionando morbimortalidad elevada ${ }^{1,2}$. El Streptococcus pneumoniae es el principal agente causal de neumonía adquirida en la comunidad (NAC), cursa frecuentemente con bacteriemia, con una incidencia estimada de 10 casos por 100.000 personas-año ${ }^{3-5}$. A comienzos del siglo XX, la mortalidad de la neumonía neumocócica era cercana a $80 \%$ y con la introducción de la antibioterapia descendió a alrededor de $20 \% 6$. Desde entonces, la letalidad de la neumonía neumocócica no se ha modificado en forma significativa ${ }^{7-10}$.

Aunque la neumonía neumocócica bacteriémica ha sido ampliamente estudiada, persisten aún algunas incógnitas, tales como las diferencias en las tasas de mortalidad comunicadas en distintas áreas geográficas y el fracaso de la terapia antibiótica para modificar la elevada mortalidad inicial ${ }^{7-11}$. Los estudios que han examinado el espectro clínico de la neumonía neumocócica bacteriémica se han focalizado en el ámbito hospitalario y unidades de pacientes crónicos, o el seguimiento de cepas aisladas en laboratorios de referencia ${ }^{4-7,9-12}$. Ortqvist y cols destacan las diferencias de mortalidad de la neumonía neumocócica bacteriémica en Huntington, Estados Unidos de Norteamérica, (26\%) y Estocolmo, Suecia (5\%) ${ }^{7}$. La mortalidad es más elevada en las primeras $48 \mathrm{~h}$ de admisión al hospital y no se ha modificado significativamente desde la era preantibiótica ${ }^{6,12}$. De hecho, se ha comunicado que el manejo en la Unidad de Cuidados Intensivos (UCI) no disminuiría en forma significativa la mortalidad precoz ${ }^{13}$. El tratamiento de la neumonía neumocócica es simple, pero la emergencia de cepas resistentes a los antibióticos ha complicado el tratamiento de esta afección ${ }^{14,15}$.

La evaluación de la gravedad de los pacientes con neumonía comunitaria permitiría predecir la evolución de los enfermos, orientar el lugar de manejo, estudio complementario y tratamiento empírico. Para ello, se han diseñado varios índices pronósticos, tales como el índice de gravedad de la neumonía y el índice pronóstico de la Sociedad Británica de Tórax modificado (CURB-65), que son aplicados rutinariamente en la clasificación de riesgo de los pacientes con neumonía ${ }^{16-18}$.
En el presente estudio se describen las características clínicas y factores pronósticos asociados a la neumonía neumocócica del adulto adquirida en la comunidad, y se compara el cuadro clínico y la evolución de los pacientes con y sin bacteriemia.

\section{PACIENTES Y MÉTODO}

Se evaluaron 151 adultos inmunocompetentes hospitalizados por un episodio de neumonía neumocócica adquirida en la comunidad en el Hospital Clínico de la Universidad Católica entre el $1^{\circ}$ de enero de 2002 y el 31 de diciembre de 2005. Los pacientes eran mayores de 15 años y cumplían los criterios diagnósticos de neumonía comunitaria propuestos por Fang y cols ${ }^{19}$. El diagnóstico de neumonía neumocócica fue confirmado mediante un cultivo positivo para Streptococcus pneumoniae aislado de una muestra de expectoración de buena calidad $(<10$ células epiteliales y $>25$ leucocitos por campo), sangre 0 líquido pleural, o antígeno urinario de neumococo positivo (Binax Inc., Scarborough ME). Se excluyó del estudio a los pacientes hospitalizados durante el último mes previo al ingreso y a aquellos que tenían alguno de los siguientes criterios de inmunosupresión: infección por VIH, neutropenia $\left(<1.000\right.$ por $\left.\mathrm{mm}^{3}\right)$, tratamiento quimioterápico 0 inmunosupresor en los seis meses previos a la admisión y uso $\geq 20 \mathrm{mg} /$ día de prednisona o su equivalente durante los últimos tres meses. Los investigadores no participaron en el manejo de los pacientes y el estudio fue aprobado por el Comité de Ética de la Institución.

Los pacientes fueron evaluados en la admisión al hospital y se registraron los siguientes antecedentes clínico-epidemiológicos: edad, sexo, lugar de procedencia, comorbilidades relacionadas con el pronóstico 3,16-18 (cardiopatía, insuficiencia cardiaca, enfermedad pulmonar obstructiva crónica, diabetes mellitus, enfermedad neurológica crónica, neoplasia, insuficiencia renal, enfermedad hepática crónica, alcoholismo), hábito tabáquico y consumo de alcohol, uso de antibióticos previo al ingreso, cuadro clínico (fiebre, tos, expectoración mucopurulenta o hemoptoica, disnea, dolor torácico, confusión mental o escalofríos), duración de la sintomatología antes de la admisión, antecedente de hospitalización durante los últimos 12 
meses, presencia de enfermedad aguda concomitante (insuficiencia cardiaca, arritmia, isquemia miocárdica, asma bronquial o EPOC reagudizado). Se consignaron los signos vitales, examen físico y mental de ingreso, y la saturación arterial de oxígeno medida con oxímetro de pulso. El compromiso cualitativo de conciencia o confusión mental fue definido por la presencia de desorientación en tiempo, espacio o personas que no corresponden a una condición basal conocida 0 coma. Los hallazgos de la radiografía de tórax fueron descritos por un radiólogo del Departamento de Radiología, quien estaba involucrado en el estudio y desconocía el cuadro clínico de los pacientes.

Se registraron los exámenes de laboratorio solicitados al ingreso al hospital, los cuales se han asociado con un curso clínico complicado o mayor mortalidad ${ }^{16-18}$ (hemograma, pruebas hepáticas, proteína $\mathrm{C}$ reactiva, gases arteriales, glicemia, albúmina sérica, electrolitos plasmáticos, función renal), los cuales fueron utilizados en el cálculo de los índices de gravedad descritos por Fine y cols (PSI score) ${ }^{16}$ y la Sociedad Británica de Tórax (CURB-65) ${ }^{18}$.

Durante la estadía en el hospital se consignaron los tratamientos antimicrobianos y la aparición de las siguientes complicaciones: ingreso a UCI, uso de ventilación mecánica, arritmias, insuficiencia cardíaca congestiva, isquemia miocárdica, accidente vascular encefálico, insuficiencia renal aguda, shock séptico, empiema pleural e infección extrapulmonar. El tratamiento fue considerado adecuado si al menos un antibiótico era activo contra el microorganismo aislado. Se consignó la letalidad en el hospital y en el seguimiento a 30 días después de la admisión.

Evaluación microbiológica. Las muestras de sangre fueron inoculadas en botellas BacT/Alert ${ }^{\circledR}$ (Biomerieux) e incubadas por siete días en el sistema automatizado. En las muestras de sangre que hubo desarrollo de colonias, y las muestras de expectoración y líquido pleural, se realizó tinción de Gram y fueron sembradas para cultivo en medio de agar sangre, chocolate y MacConkey e incubadas durante $48 \mathrm{~h}$ a $37^{\circ} \mathrm{C}$. La identificación bacteriana y los estudios de susceptibilidad se realizaron según métodos convencionales. La sensibilidad a penicilina y cefotaxima fue evaluada midiendo la concentración inhibitoria mínima (CIM) por técnica de epsilometría (E-test,) en agar Mueller-Hinton con 5\% de sangre de cordero, siguiendo las recomendaciones del Comité Nacional de Estandarización de los Laboratorios Clíni$\cos (\text { NCCLS })^{20}$. Para el control de calidad se utilizó la cepa de referencia de Streptococcus pneumoniae ATCC 49619.

Estadística. Los resultados fueron expresados como valores promedio \pm desviación estándar para las variables medidas en escala numérica y en porcentaje para las medidas en escala nominal. Las variables cualitativas fueron comparadas mediante la prueba de Chi cuadrado y las variables continuas con la prueba t de Student. Para ello se utilizaron los programas Epi-Info 6.0 (CDC, Atlanta) y SPSS 7.0 (SPSS Inc., Chicago). Los factores pronósticos medidos en la admisión fueron sometidos a análisis univariado y multivariado en un modelo de regresión logística (modalidad stepwise) que permite el control simultáneo de múltiples factores. Los parámetros que no agregaron valor predictivo no fueron retenidos en el modelo. Se calcularon los odds ratio e intervalos de confianza (IC) para el 95\%. Las diferencias entre las variables fueron consideradas significativas con un valor de $\mathrm{p}<0,05$.

\section{RESULTADOS}

Características generales de la población. En el período del estudio, se evaluaron en forma prospectiva 151 adultos inmunocompetentes hospitalizados por neumonía neumocócica adquirida en la comunidad. La edad promedio fue $64 \pm 18$ años (rango: 16-92), 58\% eran varones, 75\% tenía comorbilidad (especialmente cardiovascular, respiratoria y neurológica crónica). El diagnóstico de la infección neumocócica fue confirmado mediante cultivo de sangre ( $\mathrm{n}=73$ ), expectoración $(\mathrm{n}=61$ ), líquido pleural $(\mathrm{n}=4)$ o antígeno urinario ( $\mathrm{n}=20)$. El 16\% de los pacientes había recibido tratamiento antibiótico antes de ingresar al hospital, siendo menor el rendimiento de los hemocultivos en este grupo $(20,8 \%$ vs $53,5 \%, p<0,01)$.

La duración media de los síntomas antes del ingreso al hospital fue 6,0 $\pm 4,8$ días. Los principales síntomas reportados fueron tos (85\%), expec- 
toración $(74 \%)$, fiebre $(75 \%)$, disnea $(68 \%)$, escalofríos (34\%) y dolor torácico (32\%). Los principales hallazgos en los exámenes de laboratorio fueron hipoxemia, hipoalbuminemia, nitrógeno ureico sérico elevado y leucocitosis. La estadía media en el hospital fue $8,5 \pm 6,8$ días (rango: 1-54), la letalidad en el hospital fue $8,6 \%$ y en el seguimiento a 30 días subió a 11,3\%. La mayoría de los pacientes (88\%) fallecieron después del tercer día de admisión y su estadía en el hospital fue más prolongada $(18,8 \pm 16,2$ vs $7,6 \pm 4,1$ días en los sobrevivientes; $\mathrm{p}<0,05$ ).

La Tabla 1 resume los principales hallazgos clínicos y radiográficos de los pacientes bacteriémicos y no bacteriémicos. No hubo diferencias significativas en el cuadro clínico, evolución y mortalidad entre ambos grupos. Sin embargo, en los pacientes bacteriémicos fue más frecuente la presencia de nitrógeno ureico sérico elevado (63,0\% vs $43,4 \%$; $<0,05)$ y creatininemia elevada ( $50,7 \%$ vs $33,3 \%$; $p$ $\varangle 0,05$ ) en la admisión al hospital.

Evolución clínica y tratamiento en el hospital. La mayoría de los pacientes (96,7\%) fueron tratados con cefalosporinas de segunda o tercera generación (cefuroxima, cefotaxima, ceftriaxona) vía intravenosa como única terapia, o asociado a macrólidos (eritromicina, claritromicina; 29,8\%) o fluoroquinolonas (levofloxacina, moxifloxacina; $7,2 \%$ ). La duración del tratamiento antibiótico fluctuó entre 10 y 14 días. En esta serie no hubo pacientes tratados con penicilina o amoxicilina.

Tabla 1. C aracterísticas clínicas de los pacientes hospitalizados por neumonía neumocócica adquirida en la comunidad con y sin bacteriemia

\begin{tabular}{|c|c|c|c|}
\hline C aracterísticas & N AC bacteriémica & NAC no bacteriémica & Total \\
\hline $\mathrm{n}$ & 73 & 78 & 151 \\
\hline Edad (años) & $66,0 \pm 17,9$ & $62,6 \pm 18,7$ & $64,2 \pm 18,4$ \\
\hline Masculino & 45 & 42 & 87 \\
\hline Fumador activo & 15 & 18 & 33 \\
\hline \multicolumn{4}{|l|}{ Comorbilidades } \\
\hline$\geq 1$ comorbilidad & 55 & 59 & 114 \\
\hline Enfermedad cardiovascular & 31 & 24 & 55 \\
\hline Enfermedad respiratoria crónica & 18 & 30 & 48 \\
\hline Diabetes mellitus & 11 & 16 & 27 \\
\hline Enfermedad neurológica crónica & 8 & 5 & 13 \\
\hline \multicolumn{4}{|l|}{ Cuadro clínico } \\
\hline Tos & 61 & 67 & 128 \\
\hline Expectoración & 52 & 60 & 112 \\
\hline Disnea & 46 & 57 & 103 \\
\hline Dolor torácico & 28 & 21 & 49 \\
\hline Fiebre & 58 & 55 & 113 \\
\hline Escalofríos & 27 & 25 & 52 \\
\hline \multicolumn{4}{|l|}{ Indice de Fine } \\
\hline Clases I-II-III & 28 & 40 & 68 \\
\hline Clases IV-V & 45 & 38 & 83 \\
\hline NAC multilobar & 21 & 16 & 37 \\
\hline Derrame pleural & 13 & 12 & 25 \\
\hline Admisión a UCI & 20 & 19 & 39 \\
\hline Estadía en el hospital (días) & $8,9 \pm 6,1$ & $8,2 \pm 7,6$ & $8,5 \pm 6,8$ \\
\hline Letalidad en el hospital & 6 & 7 & 13 \\
\hline Letalidad a 30 días & 8 & 9 & 17 \\
\hline
\end{tabular}

Nota: NAC: neumonía comunitaria. Los resultados se expresan como valores promedio \pm DE o número de casos. 
Todos los pacientes recibieron tratamiento antibiótico apropiado, con cobertura antineumocócica, en la admisión al hospital. Ningún esquema antibiótico prescripto se asoció a mayor riesgo de complicaciones o mortalidad en el hospital.

El estudio de susceptibilidad a antimicrobianos estuvo disponible para 71 de las 73 cepas de neumococo aisladas de hemocultivos. Se aislaron tres cepas resistentes a penicilina (CIM $\geq 2 \mu \mathrm{g} / \mathrm{mL}$ y menor de $4 \mu \mathrm{g} / \mathrm{mL}$ ), cuatro cepas resistentes a eritromicina y una cepa resistente a cefalosporinas de tercera generación. La enfermedad fue menos severa en los pacientes con infección neumocócica resistente a antimicrobianos (PSI score en la admisión $89,7 \pm 48,5$ vs $106,5 \pm 37,7, \mathrm{p}=0,18$; mortalidad 0 vs $13,5 \%, p=0,17$ ).

Cuarenta y tres pacientes $(28,4 \%)$ desarrollaron alguna complicación durante su permanencia en el hospital y seguimiento a 30 días, siendo similar en los pacientes bacteriémicos (26\%) y no bacteriémi$\cos (30,8 \%)$. Las principales complicaciones en la evolución, tales como insuficiencia respiratoria grave que requirió ventilación mecánica, shock séptico, insuficiencia renal, empiema, insuficiencia cardiaca congestiva y arritmias, afectaron en forma similar a ambos grupos. El 26\% de los pacientes con neumonía neumocócica fueron admitidos a la UCI por insuficiencia respiratoria grave (86\%), necesidad de conexión a ventilación mecánica (33\%) o compromiso hemodinámico (21\%).

Cuatro pacientes fallecieron durante la primera semana en el hospital, y el deceso fue atribuible a shock séptico $(\mathrm{n}=2)$ o falla respiratoria aguda $(\mathrm{n}=2)$. El lugar de ingreso, la estadía en el hospital y mortalidad fueron similares en los pacientes con y sin bacteriemia. En la Tabla 2 se describe la relación entre los índices pronósticos (índice de Fine y CURB-65) y la mortalidad en el seguimiento a 30 días. El valor predictivo de ambos índices pronósticos fue aceptable, el área bajo la curva fue 0,80 para el índice de Fine (IC 95\% 0,71-0,89; $p<0,001$ ) y 0,69 para el CURB-65 (IC 95\% 0,55-0,84; $\mathrm{p}<0,005$ ). Un tercio de los pacientes bacteriémicos correspondieron a las categonías de bajo riesgo del índice de Fine y ninguno falleció en el hospital.

Factores pronósticos. Dos tercios de los fallecidos eran adultos mayores de 70 años. Las categonías de riesgo IV-V de Fine, CURB-65 $\geq 2$ puntos, la admisión a UCI y necesidad de ventilación mecánica se asociaron a mayor riesgo de morir en el seguimiento a 30 días. El índice de gravedad de Fine fue más elevado en los pacientes con bacteriemia $(104 \pm 40$ vs $90 \pm 34, \mathrm{p}$ $\varangle 0,05)$. La letalidad de las categonáas de bajo riesgo (clases I a III) fue 1,5\% comparado con 19,2\% en las categonías de riesgo elevado (clases IV-V). Ambos índices permitieron pesquisar a los pacientes de resgo elevado, pero el índice de Fine fue más certero en la pesquisa de pacientes de bajo riesgo (Tabla 2).

Los factores asociados a mayor riesgo de muerte en el seguimiento a 30 días fueron: edad avanzada ( $\geq 75$ años), neoplasia activa, confusión mental, hipotensión arterial, ausencia de fiebre o calofińos, disfunción renal (nitrógeno ureico sérico mayor de $30 \mathrm{mg} /$

Tabla 2. Letalidad de la neumonía neumocócica bacteriémica y no bacteriémica según categorías de riesgo

\begin{tabular}{|lccc|}
\hline Categorías de riesgo & $\begin{array}{c}\text { Bacteriémicos } \\
(\mathbf{n}=\mathbf{7 3}) \\
\mathbf{n} \text { muertes/pacientes }\end{array}$ & $\begin{array}{c}\text { No-bacteriémicos } \\
(\mathbf{n}=\mathbf{7 8}) \\
\mathbf{n} \text { muertes/ pacientes }\end{array}$ & $\begin{array}{c}\text { Total } \\
\mathbf{( n = 1 5 1 )} \\
\mathbf{n} \text { muertes / pacientes }\end{array}$ \\
\hline Indice de Fine16 & & & \\
Clases I-II-III & $0 / 28$ & $1 / 40$ & $1 / 68$ \\
Clase IV & $2 / 27$ & $6 / 31$ & $8 / 58$ \\
Clase V & $6 / 18$ & $2 / 7$ & $8 / 25$ ** \\
CURB-6518 & $0 / 25$ & $3 / 35$ & $3 / 60$ \\
0 - 1 punto & $0 / 19$ & $3 / 20$ & $3 / 39$ \\
2 puntos & $8 / 29$ & $3 / 23$ & $11 / 52 *$ \\
$\geq 3$ puntos & & & \\
\hline
\end{tabular}

$*_{p}<0,05$ y **p $<0,001$ comparado con la categoría de bajo riesgo. 
dL o creatininemia $\geq 1,3 \mathrm{mg} / \mathrm{dL}$ ), hipematremia ( $\geq 145$ $\mathrm{mEq} / \mathrm{L}$ ), acidosis metabólica ( $\mathrm{pH}$ arterial $\leq 7,33 \mathrm{y}$ bicarbonato sérico $\leq 19 \mathrm{mEq} / \mathrm{L}$ ), hipoalbuminemia $(\leq 2,8 \mathrm{~g} / \mathrm{dL})$, admisión a UCI y necesidad de ventilación mecánica (Tabla 3). El riesgo de muerte fue más elevado en los pacientes con cuadro clínico breve ( $\leq 3$ días). El uso de antibióticos antes del ingreso no se relacionó con el riesgo de complicaciones o muerte en el hospital.

Los factores asociados a mayor riesgo de muerte en el análisis multivariado fueron hipotensión arterial diastólica, nitrógeno ureico sérico elevado y necesidad de ventilación mecánica por falla respiratoria grave (Tabla 4).

\section{Discusión}

Evaluamos el cuadro clínico, tratamiento y factores de riesgo de muerte en adultos inmunocompetentes hospitalizados por un episodio de neumonía neumo- cócica adquinida en la comunidad. Es el estudio más extenso que se ha realizado en nuestro medio sobre este tópico específico, donde abarcamos cuatro años para aumentar el tamaño muestral y evitar los factores estacionales, medimos en forma prospectiva dos índices de riesgo validados en la literatura (índice de Fine y CURB-65) ${ }^{16-18}$ para caracterizar a nuestra población, y excluimos a los pacientes con infección neumocócica nosocomial.

En nuestro estudio, $48 \%$ de los pacientes evaluados tenía infección neumocócica bacteriémica, similar a lo comunicado por Schaaf y cols (64/ $105)^{21}$ y superior a lo descrito por Burman y cols ${ }^{22}$ (21\%), quienes utilizaron varias técnicas serológicas para diagnosticar la infección neumocócica. En un estudio realizado en 2.776 pacientes hospitalizados por neumonía comunitaria en los condados de Franklin y Summit, Ohio, en 1991, la tasa de NAC neumocócica bacteriémica fue $5,5 \%{ }^{23}$. El número de pacientes con neumonía neumocócica identificados en los diferentes estudios varía de acuerdo

Tabla 3. Factores pronósticos en 151 adultos inmunocompetentes hospitalizados por neumonía neumocócica adquirida en la comunidad. Análisis univariado

\begin{tabular}{|c|c|c|c|c|c|}
\hline Variables & Pacientes & Fallecidos & 0 dds Ratio & IC $95 \%$ & p \\
\hline Edad $\geq 75$ años & 55 & 11 & 3,8 & $1,2-12,3$ & 0,01 \\
\hline Neoplasia activa & 17 & 5 & 4,2 & $1,3-14,0$ & 0,018 \\
\hline Duración síntomas $\leq 3 \mathrm{~d}$ & 48 & 10 & 3,2 & $1,1-9,1$ & 0,021 \\
\hline Fiebre & 113 & 9 & 0,3 & $0,1-0,9$ & 0,033 \\
\hline Escalofríos & 52 & 2 & 0,2 & $0,1-0,9$ & 0,05 \\
\hline Confusión mental & 21 & 7 & 6,0 & $2,0-18,2$ & 0,002 \\
\hline $\mathrm{PAS} \leq 95 \mathrm{mmHg}$ & 27 & 7 & 3,9 & $1,3-11,5$ & 0,009 \\
\hline $\mathrm{PAD} \leq 50 \mathrm{mmHg}$ & 18 & 6 & 5,5 & $1,7-17,3$ & 0,002 \\
\hline Temp $<37,5^{\circ} \mathrm{C}$ & 78 & 14 & 5,0 & $1,4-18,0$ & 0,008 \\
\hline $\mathrm{BUN}>30 \mathrm{mg} / \mathrm{dL}$ & 42 & 13 & 11,5 & $3,5-38,1$ & 0,001 \\
\hline Creatinina $\geq 1,3 \mathrm{mg} / \mathrm{dL}$ & 53 & 11 & 3,9 & $1,4-11,3$ & 0,008 \\
\hline $\mathrm{Na}^{+} \geq 145 \mathrm{mEq} / \mathrm{L}$ & 6 & 3 & 9,5 & $1,7-51,7$ & 0,002 \\
\hline $\mathrm{pH}$ arterial $\leq 7,33$ & 6 & 3 & 8,0 & $1,5-43,5$ & 0,006 \\
\hline $\mathrm{PaO}_{2} / \mathrm{FiO}_{2}<200$ & 41 & 11 & 5,0 & $1,7-14,7$ & 0,002 \\
\hline $\mathrm{HCO}_{3} \leq 19 \mathrm{mEq} / \mathrm{L}$ & 23 & 7 & 4,3 & $1,4-12,8$ & 0,006 \\
\hline Albúmina $\leq 2,8 \mathrm{~g} / \mathrm{dL}$ & 27 & 7 & 4,4 & $1,5-13,0$ & 0,005 \\
\hline Admisión a UCI & 39 & 10 & 5,2 & $1,8-14,8$ & 0,001 \\
\hline Ventilación mecánica & 13 & 8 & 22,9 & $6,2-84,6$ & 0,001 \\
\hline Shock & 17 & 11 & 39,1 & $10,8-141$ & 0,001 \\
\hline Insuficiencia renal & 13 & 6 & 9,9 & $2,8-34,6$ & 0,001 \\
\hline Estadía en hospital $>12 \mathrm{~d}$ & 27 & 9 & 7,3 & $2,5-21,2$ & 0,001 \\
\hline
\end{tabular}

Nota: IC 95\%: intervalo de confianza de 95\%, PAS: presión arterial sistólica, PAD: presión arterial diastólica, Temp: temperatura axilar, BUN: nitrógeno ureico sérico, $\mathrm{HCO}_{3}$ : bicarbonato sérico, UCI: unidad de cuidado intensivo. 
Tabla 4. Factores pronósticos en adultos inmunocompetentes hospitalizados por neumonía neumocócica adquirida en la comunidad. Análisis multivariado

\begin{tabular}{|lccccl|}
\hline Variables & Pacientes & Fallecidos & Odds ratio & IC 95\% & p \\
\hline BUN $>30 \mathrm{mg} / \mathrm{dL}$ & 42 & 13 & 6,8 & $1,8-26,0$ & 0,005 \\
Ventilación mecánica & 13 & 8 & 7,4 & $1,7-31,8$ & 0,007 \\
PAD $\leq 50 \mathrm{mmHg}$ & 18 & 6 & 3,9 & $1,0-17,0$ & 0,05 \\
\hline
\end{tabular}

Nota: IC 95\%: intervalo de confianza de 95\%, BUN: nitrógeno ureico sérico, PAD: presión arterial diastólica.

con las técnicas microbiológicas empleadas (serología, detección de antígenos, cultivos), y la rigurosidad con que se implementan las diferentes técnicas y procedimientos microbiológicos.

En nuestra serie, la letalidad de la neumonía neumocócica adquirida en la comunidad del adulto fue $11,3 \%$ en el seguimiento a 30 días; similar a lo descrito por Ortqvist $(7 \%)^{5}$, Díaz $(13 \%)^{11}$, Frankel $(12 \%)^{25}$, Espósito $(13 \%)^{26}$ y Vila-Corcoles $(15,4 \%)^{27}$. El cuadro clínico, la evolución y pronóstico fueron similares en pacientes con y sin bacteriemia, lo cual había sido descrito por Ramírez y cols ${ }^{28}$.

La edad avanzada se asoció a mayor riesgo de muerte en el hospital, lo cual ha sido mencionado en otros estudios $3,11,16$, y entre las comorbilidades, la presencia de neoplasia activa constituyó factor de mal pronóstico. La importancia de las enfermedades crónicas en el pronóstico de la enfermedad neumocócica ha sido destacada en múltiples estudios, sin embargo en la mayoría no se realizó análisis multivariado. En un metaanálisis de factores pronósticos en neumonía comunitaria del adulto $^{3}$, la enfermedad neurológica crónica (OR: 4,6), neoplasia activa (OR: 2,8) e insuficiencia cardiaca congestiva (OR: 2,4) constituyeron factores de mal pronóstico en la evolución de los enfermos.

En el análisis multivariado, la hipotensión arterial, disfunción renal y uso de ventilación mecánica fueron

\section{REFERENCIAS}

1. MARRIE TJ. Bacteraemic pneumococcal pneumonia: a continuously evolving disease. J Infect 1992; 24: 247-55.

2. Afessa B, Greaves WL, FREDERICK WR. Pneumococcal bacteremia in adults: a 14-year experience in an innercity university hospital. Clin Infect Dis 1995; 21: 345-51.

3. Fine MJ, Smith MA, Carson CA, Mutha SS, Sankey SS, Weissfeld LA, Kapoor WN. Prognosis and outcomes of patients with community-acquired pneumonia. A predictores independientes de mal pronósti$\mathrm{Co}^{3,8,11,16,18}$. La edad avanzada, hipotensión arterial y nitrógeno ureico sérico elevado están incluidos en los índices pronósticos compuestos recomendados en las Guías Clínicas de Neumonía patrocinadas por la Sociedad Americana de Tórax (índice de gravedad de la neumonía) y Sociedad Británica de Tórax (CURB$65)^{29-31}$. Ambas reglas predictivas han demostrado ser excelentes predictores pronósticos. Las categonás de bajo riesgo descritas por Fine y cols detectaron a los pacientes hospitalizados por neumonía neumocócica de bajo riesgo de complicaciones y muerte (menor de $2 \%)$; mientras que el índice pronóstico de la BTS modificado permitió pesquisar mejor a los pacientes de riesgo elevado ( $\geq 3$ puntos). En nuestra serie, un tercio de los pacientes con neumonía neumocócica bacteriémica estaban en las categonías de bajo riesgo de Fine y ninguno falleció en el seguimiento a 30 días, hallazgo similar fue descrito por Brandenburg y $\operatorname{cols}^{32}$.

En conclusión, los índices de gravedad propuestos en las guías clínicas extranjeras ${ }^{29-31}$ permiten predecir el riesgo de muerte en adultos hospitalizados con neumonía neumocócica en nuestro medio. La hipotensión arterial (OR: 3,9), nitrógeno ureico sérico elevado (OR: 6,8) y la necesidad de ventilación mecánica por falla respiratoria (OR: 7,4) se asociaron a mayor riesgo de muerte.

meta-analysis. JAMA 1996; 275: 134-41.

4. Burman LA, Norrby R, Trolfors B. Invasive pneumococcal infections: incidence, predisposing factors, and prognosis. Rev Infect Dis 1985; 7: 133-42.

5. Ortquist A, Grepe A, Julander I, Kalin M. Bacteremic pneumococcal pneumonia in Sweden: clinical course and outcome and comparison with non-bacteremic pneumococcal and mycoplasmal pneumonias. Scand J Infect Dis 1988; 20: 163-71.

6. Austrian R, Gold J. Pneumococcal bacteremia with 
especial reference to bacteremic pneumococcal pneumonia. Ann Intern Med 1964; 60: 759-76.

7. Ortovist A, Kalin M, Julander I, Mufson MA. Deaths in bacteremic pneumococcal pneumonia. A comparison of two populations - Huntington, West Virginia, and Stockholm, Sweden. Chest 1993; 103: 710-6.

8. GILBERT K, Fine MJ. Assessing prognosis and predicting patient outcomes in community-acquired pneumonia. Semin Respir Infect 1994; 9: 140-52.

9. Mirzanejad Y, Roman S, Talbot J, Nicolle L. Pneumococcal bacteremia in two tertiary care hospitals in Winnipeg, Canada. The Pneumococcal Bacteremia Study Group. Chest 1996; 109: 173-8.

10. Plouffe JF, Breiman RF, Fackiam RR. Bacteremia with Streptococcus pneumoniae. Implications for therapy and prevention. Franklin County Pneumonia Study Group. JAMA 1996; 275: 194-8.

11. Díaz A, Torres C, Flores LJ, García P, Saddías F. Community acquired pneumococcal pneumonia in hospitalized adult patients. Rev Méd Chile 2003; 131: 505-14.

12. Kramer MR, Rudensky B, Hadas-Halperin I, Isacsohn M, Meizer E. Pneumococcal bacteremia - no change in mortality in 30 years: analysis of 104 cases and review of the literature. Isr J Med Sci 1987; 23: 174-80.

13. Hook EW III, HorTON CA, SCHABERG DR. Failure of intensive care unit support to influence mortality from pneumococcal bacteremia. JAMA 1983; 249: 1055-7.

14. Pallares R, Lñares J, Vadilo $M$, Cabellos $C$, Manresa $F$, VILADRICH PF ET AL Resistance to penicillin and cephalosporin and mortality from severe pneumococcal pneumonia in Barcelona, Spain. NEngl J Med 1995; 333: 474-80.

15. Hofmann J, Cetron MS, FarLey MM, Baughman WS, FACKIAM RR, ELUOT JA ET AL. The prevalence of drugresistant Streptococcus pneumoniae in Atlanta. N Engl J Med 1995; 333: 481-6.

16. Fine MJ, Auble TE, Yealy DM, Hanusa BH, Weissfeld LA, SiNGER DE ET AL. A prediction rule to identify lowrisk patients with community-acquired pneumonia. N Engl J Med 1997; 336: 243-50.

17. Harrison BD, Farr BM, Pugh S, SelKon JB. Communityacquired pneumonia in adults in British hospitals in 1982-1983: a survey of aetiology, mortality, prognostic factors and outcome. Q J Med 1987; 62: 195-220.

18. Lim WS, Van Der Eerden MM, Laing R, Boersma WG, Karalus N, Town GI ET AL. Defining community acquired pneumonia severity on presentation to hospital: an international derivation and validation study. Thorax 2003; 58: 377-82.

19. Fang GD, Fine M, Orloff J, Arisumi D, Yu VL, Kapoor W ET AL. New emerging etiologies for communityacquired pneumonia with implications for therapy. A prospective multicenter study of 359 cases. Medicine (Baltimore) 1990; 69: 307-16.

20. National Committee for Cunical Laboratory Standards (NCCLS). Performance standards for antimicrobial susceptibility testing- $14^{\text {th }}$ informational supplement, approved standard M 100-S14. Wayne County, NCCLS, 2004.
21. SchaAf B, Kruse J, Rupp J, Reinert RR, Droemann D, ZABEL P ET AL. Sepsis severity predicts outcome in community-acquired pneumococcal pneumonia. Eur Respir J 2007; 30: 517-24.

22. Burman LA, Trowfors B, Andersson B, Henrichsen J, Juto P, KAUNGS I ET AL. Diagnosis of pneumonia by cultures, bacterial and viral antigen detection tests, and serology with special reference to antibodies against pneumococcal antigens. J Infect Dis 1991; 163: 1087-93.

23. Marston BJ, Plouffe JF, File TM Jr, Hackman BA, SALSTROM SJ, LipMAN HB ET aL. Incidence of community-acquired pneumonia requiring hospitalization. Results of a population-based active surveillance study in Ohio. Arch Intern Med 1997; 157: 1709-18.

24. WATANAKUNAKORN C, BAILEY TA. Adult bacteremic pneumococcal pneumonia in a community teaching hospital, 1992-96. A detailed analysis of 108 cases. Arch Intern Med 1997; 157: 1965-71.

25. Frankel RE, Virata M, Hardalo C, Altice FL, Friediand G. Invasive pneumococcal disease: clinical features, serotypes, and antimicrobial resistance patterns in cases involving patients with and without human immunodeficiency virus infection. Clin Infect Dis 1996; 23: 577-84.

26. Espósito AL. Community-acquired bacteremic pneumococcal pneumonia. Effect of age on manifestations and outcome. Arch Intern Med 1984; 144: 945-8.

27. Vila-Corcoles A, Bejarano-Romero F, Salsench E, OchoaGondar O, De Diego C, Gómez-Bertomeu F et al. Drugresistance in Streptococcus pneumoniae isolates among Spanish middle aged and older adults with communityacquired pneumonia. BMC Infect Dis 2009; 9: 36.

28. Bordón J, Peyrani P, Brock GN, Blasi F, Rello J, File T, RAmírez J, CAPO Study Group. The presence of pneumococcal bacteremia does not influence clinical outcomes in patients with community-acquired pneumonia: results from the Community-Acquired Pneumonia Organization (CAPO) International Cohort study. Chest 2008; 133: 618-24.

29. Mandell LA, Wunderink RG, Anzueto A, Bartlett JG, CAMPBELl GD, DeAN NC ET AL. Infectious Diseases Society of America/American Thoracic Society Consensus Guidelines on the management of community-acquired pneumonia in adults. Clin Infect Dis 2007; 44 (Suppl 2): S27-72.

30. British Thoracic Society Standards of Care Committee. British Thoracic Society Guidelines for the management of community-acquired pneumonia in adults. Thorax 2001; 56 (Suppl IV): 1-64.

31. British Thoracic Society Pneumonia Guidelines CommitTEE. The British Thoracic Society Guidelines for the management of community-acquired pneumonia in adults - 2009 update. www.brit-thoracic.org.uk/guidelines.

32. Brandendurg JA, Marrie TJ, Coley CM, Singer DE, Obrosky DS, KAPOOR WN ET AL Clinical presentation, processes and outcomes of care for patients with pneumococcal pneumonia. J Gen Intern Med 2000; 15: 638-46. 